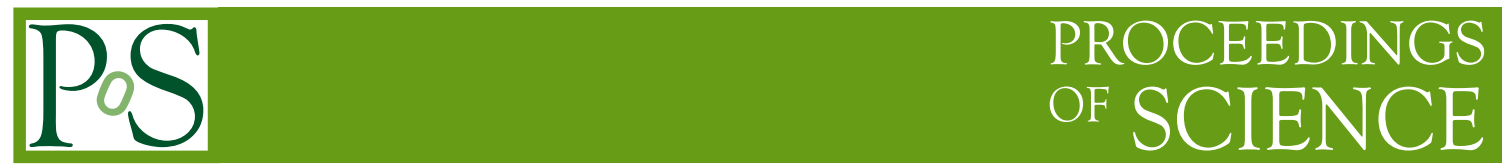

\title{
QCD with massive gluons
}

\section{Sergey Larin*}

Institute for Nuclear Research, Moscow

E-mail: larindinr.ac.ru

It is shown that the Lagrangian of Quantum Chromodynamics can be modified by the adding gluon masses. On mass-shell renormalizability of the resulting theory is discussed.

XXII International Baldin Seminar on High Energy Physics Problems

15-20 September, 2014

JINR, Dubna, Russia

${ }^{*}$ Speaker. 
The discovery [1] of asymptotic freedom in Quantum Chromodynamics (QCD) has lead to the establishment of QCD as the theory of strong interactions. The gauge bosons of the theory, the gluons, are considered to be massless to have gauge invariance and correspondingly renormalizability. In the present paper it is shown that the QCD Lagrangian should be modified by the adding gluon masses to ensure that QCD does not contradict to experiments. On mass-shell renormalizability of the resulting theory is discussed.

The Lagrangian of QCD is

$$
\begin{gathered}
L_{Q C D}=-\frac{1}{4} F_{\mu \nu}^{a} F_{\mu \nu}^{a}+i \bar{\psi}_{f} \gamma_{\mu} D_{\mu} \psi_{f}-m_{f} \bar{\psi}_{f} \psi_{f} \\
-\frac{1}{\xi}\left(\partial^{\mu} A_{\mu}^{a}\right)^{2}+\partial^{\mu} \bar{c}^{a}\left(\partial_{\mu} c^{a}-g f^{a b c} c^{b} A_{\mu}^{c}\right)+\text { counterterms }
\end{gathered}
$$

where $F_{\mu \nu}^{a}=\partial_{\mu} A_{v}^{a}-\partial_{v} A_{\mu}^{a}+g f^{a b c} A_{\mu}^{b} A_{v}^{c}$ is the gluon field strength tensor, $D_{\mu}=\partial_{\mu}-i g A_{\mu}^{a} T^{a}$ is the covariant derivative. The quark fields $\psi_{f}$ transform as the fundamental representation of the colour group $S U(3), f=u, d, s, c, b, t$ is the flavour index. The gluons $A_{\mu}^{a}$ transform as the adjoint representation of this group. $c^{a}$ are the ghost fields, $\xi$ is the gauge parameter of the usually chosen general covariant gauge, $f^{a b c}$ are the structure constants of the group, $T^{a}$ are the generators of the fundamental representation. $g=g(\mu)$ is the renormalized strong coupling constant, $g^{2} /\left(16 \pi^{2}\right) \equiv$ $a_{s}, m_{f}=m_{f}(\mu)$ is the Lagrangian (renormalized) mass of a quark with a flavor $f$, and $\mu$ is the renormalization point. The summations over repeated indexes are assumed.

Let us consider the vacuum polarization function $\Pi\left(q^{2}\right)$

$$
\left(-q^{2} g_{\mu v}+q_{\mu} q_{v}\right) \Pi\left(q^{2}\right)=i \int \mathrm{d} x e^{i q x}\left\langle 0\left|T j_{\mu}(x) j_{v}(0)\right| 0\right\rangle .
$$

where $j_{\mu}=\sum_{f} q_{f} \bar{\psi}_{f} \gamma_{\mu} \psi_{f}$ is the electromagnetic quark current and $q_{f}=2 / 3,-1 / 3, \ldots$ is the electromagnetic charge of the quark with a flavor $f$.

According to general principles of local quantum field theory the function $\Pi\left(q^{2}\right)$ satisfies the Källen-Lehmann [2] spectral representation

$$
\Pi\left(q^{2}\right)=\frac{1}{12 \pi^{2}} \int_{4 m_{\pi}^{2}}^{\infty} d s \frac{R(s)}{s-q^{2}-i 0},
$$

where the ratio $R(s)=\sigma_{\text {tot }}\left(e^{+} e^{-} \rightarrow\right.$ hadrons $) / \sigma\left(e^{+} e^{-} \rightarrow \mu^{+} \mu^{-}\right)$is the normalized total crosssection of electron-positron annihilation into hadrons, $m_{\pi}$ is a pion mass.

The Källen-Lehmann representation determines the analytic properties of $\Pi\left(q^{2}\right)$ which should be an analytic function in the complex $q^{2}$-plane with the cut starting from the first physical threshold, i.e. as it is dictated by experiments from the two-pion threshold $q^{2}=4 m_{\pi}^{2}$. In particular, one gets for the discontinuity of $\Pi\left(q^{2}\right)$ on the cut

$$
\Delta \Pi\left(q^{2}\right) \equiv \Pi\left(q^{2}+i 0\right)-\Pi\left(q^{2}-i 0\right)=\left\{\begin{array}{cc}
i R\left(q^{2}\right) /(6 \pi) & \text { at } s>4 m_{\pi}^{2} \\
0 & \text { at } s<4 m_{\pi}^{2} .
\end{array}\right.
$$

Perturbative QCD produces the following expression for the discontinuity

$$
\Delta \Pi\left(q^{2}\right)_{p Q C D}=\theta\left(q^{2}\right) \rho_{\text {gluon }}\left(q^{2}\right)+\theta\left(q^{2}-4 M_{u}^{2}\right) \rho_{\text {quark }}\left(q^{2}\right) .
$$


The gluon spectral density $\rho_{\text {gluon }}\left(q^{2}\right)$ contributes for $q^{2}>0$ as it is indicated by the theta-function $\theta\left(q^{2}\right)$. This is the known zero threshold. It arises from those absorptive parts of Feynman diagrams of $\Pi\left(q^{2}\right)$ which are produced by purely gluonic cuts of the diagrams (i.e. by the Cutcosky cuts which cross only gluon propagators of diagrams). As it is well known such diagrams appear for the first time at the four-loop level in the order $a_{s}^{3}$ (corresponding cuts cross 3 gluon propagators).

The quark spectral density $\rho_{\text {quark }}\left(q^{2}\right)$ arises from the quark cuts of the diagrams (i.e. from the cuts which cross two or more quark propagators of the diagrams). It contributes for $q^{2}>4 M_{u}^{2}$ where $M_{u}$ is the perturbative pole mass of the lightest $u$-quark, defined as the pole of the quark propagator within perturbation theory. A perturbative quark pole mass

$$
M_{f}=m_{f}(\mu)+O\left(a_{s}\right)
$$

appears after summation of perturbative corrections to a quark propagator. It is a renormalization group invariant quantity, i.e. independent on the renormalization point $\mu$ and on the choice of the subtraction scheme. In this sense it behaves as a physical object and that is why it is natural to use this definition of a quark mass to parametrize the theory.

We will not discuss here the important by themselves questions of convergence or divergence of corresponding perturbative QCD series at low or at high energies. Here we will just accept that our conventional perturbation theory is adequate to the exact solution of the theory, i.e. it correctly reproduces the perturbative expansion of the exact solution.

Hence one gets within QCD that $\Delta \Pi\left(q^{2}\right)$ is non-zero in the energy interval $0<q^{2}<4 m_{\pi}^{2}$ since the perturbative contribution $\Delta \Pi\left(q^{2}\right)_{p Q C D}$ is non-zero in this interval. And we would like to stress here that one should get in QCD an exact zero below the two-pion threshold as it is dictated by experiments. There are of course also non-perturbative contributions, i.e. contributions of the type of $e^{-1 / a_{s}}$ which are invisible in the perturbative expansion at $a_{s}=0_{+}$

$$
e^{-1 / a_{s}}=0 \cdot a_{s}+0 \cdot a_{s}^{2}+\ldots
$$

At this point it is interesting to see on simple examples how non-perturbative contributions can be separated from perturbative ones. We consider first the following function

$$
\frac{1}{e^{a_{s}}+e^{-1 / a_{s}}}
$$

which contains both perturbative and non-perturbative contributions.

Let us nullify in this function the non-perturbative object $e^{-1 / a_{s}}$. Then we are left with the purely perturbative function $\frac{1}{e^{a_{s}}}$. Let us now subtract and add this function to the original one:

$$
\frac{1}{e^{a_{s}}+e^{-1 / a_{s}}} \equiv \frac{1}{e^{a_{s}}+e^{-1 / a_{s}}}-\frac{1}{e^{a_{s}}}+\frac{1}{e^{a_{s}}}=-\frac{e^{-1 / a_{s}}}{e^{a_{s}}+e^{-1 / a_{s}}}+e^{-a_{s}},
$$

where we combained first two terms into one. Thus we have presented the original function as the sum of the purely non-perturbative and purely perturbative terms.

Let us consider one more simple example:

$$
\sin \left(e^{a_{s}}+e^{-1 / a_{s}}\right) .
$$


We again nullify in the above function the non-perturbative object $e^{-1 / a_{s}}$ to get the purely perturbative function $\sin \left(e^{a_{s}}\right)$. Again we subtract and add this perturbative functin to the original one:

$$
\sin \left(e^{a_{s}}+e^{-1 / a_{s}}\right)=\left[\sin \left(e^{a_{s}}+e^{-1 / a_{s}}\right)-\sin \left(e^{a_{s}}\right)\right]+\sin \left(e^{a_{s}}\right) .
$$

Here in the square brackets we have the purely non-perturbative function and outside - the purely perturbative one.

The general rule is as follows. In an original function which is a mix of perturbative and non-perturbative contributions one should nullify all non-perturbative objects getting in this way a purely perturbative function. Then one should subtract and add this perturbative function to the original one. The difference of the original function and the purely perturbative one will form the purely non-perturbative contribution.

Let us now return to the analysis of the spectral dencity. We note that non-perturbative contributions can not exactly cancel the perturbative contribution in the continuous interval $0<q^{2}<4 m_{\pi}^{2}$ because of the different dependence on $a_{s}$. To get that $\Delta \Pi\left(q^{2}\right)=0$ at $0<q^{2}<4 m_{\pi}^{2}$ in agreement with experiments one should move perturbative gluon and quark thresholds above $q^{2}=4 m_{\pi}^{2}$. That is why we should introduce the non-zero Lagrangian gluon masses.

The first naive objection here is that nobody trusts perturbation theory below the two-pion threshold, i.e. that the corresponding perturbative series is heavily divergent in this energy region. But for us here only the principal existence of the pertubative series with finite coefficients below the two-pion threshold is of importance independently on the question of its divergence.

Thus one obtains the following restrictions on the (perturbative pole) masses of gluons and quarks

$$
\begin{gathered}
\left(3 M_{g l}\right)^{2}>4 m_{\pi}^{2}, \\
4 M_{u}^{2}>4 m_{\pi}^{2} .
\end{gathered}
$$

Although the restriction on $M_{u}$ seems to be quite strong for the lightest u-quark it is not excluded from the first principles.

To construct QCD with massive gluons we will follow the approach of [3]. Presently this is the only known way to get (on mass-shell) renormalizable theory of massive gluons without color scalars (color scalars are rejected by experiments). Within this approach one starts from a renormalizable theory with scalar fields using the Englert-Brout-Higgs mechanism of spontaneous symmetry breaking [4] and after transition to the unitary gauge removes remaining massive scalar fields. Thus we add to the massless QCD Lagrangian (1) the scalar part to begin with the following general Lagrangian

$$
\begin{gathered}
L_{Q C D+\text { scalars }}=-\frac{1}{4} F_{\mu \nu}^{a} F_{\mu \nu}^{a}+i \bar{\psi}_{f} \gamma_{\mu} D_{\mu} \psi_{f}-m_{f} \bar{\psi}_{f} \psi_{f}+ \\
\left(D_{\mu} \Phi\right)^{+} D_{\mu} \Phi+\left(D_{\mu} \Sigma\right)^{+} D_{\mu} \Sigma-\lambda_{1}\left(\Phi^{+} \Phi-v_{1}^{2}\right)^{2}-\lambda_{2}\left(\Sigma^{+} \Sigma-v_{2}^{2}\right)^{2} \\
-\lambda_{3}\left(\Phi^{+} \Phi+\Sigma^{+} \Sigma-v_{1}^{2}-v_{2}^{2}\right)^{2}-\lambda_{4}\left(\Phi^{+} \Sigma\right)\left(\Sigma^{+} \Phi\right) \\
+L_{g f}+L_{g c}+\text { counterterms }
\end{gathered}
$$

where we introduced two triplets $\Phi(x)$ and $\Sigma(x)$ of complex scalar fields in the fundamental representation of the $S U(3)$ color group to get all gluon massive. $L_{g f}$ is the gauge fixing part of the 
Lagrangian in some chosen gauge and $L_{g c}$ is the corresponding gauge compensating part with the Faddeev-Popov ghost fields.

We can choose the following shifts of scalar fields by the quantities $v_{1}$ and $v_{2}$ to generate masses of all eight gluons

$$
\Phi(x)=\left(\begin{array}{l}
\phi_{1}(x)+i \phi_{2}(x)+v_{1} \\
\phi_{3}(x)+i \phi_{4}(x) \\
\phi_{5}(x)+i \phi_{6}(x)
\end{array}\right), \Sigma(x)=\left(\begin{array}{l}
\sigma_{1}(x)+i \sigma_{2}(x) \\
\sigma_{3}(x)+i \sigma_{4}(x)+v_{2} \\
\sigma_{5}(x)+i \sigma_{6}(x)
\end{array}\right) .
$$

Choosing for simplicity $v_{1}=v_{2} \equiv v$ one obtains the following massive terms for gluons in the Lagrangian

$$
\begin{gathered}
L_{M}=m_{g l}^{2}\left[\left(A^{1}\right)^{2}+\left(A^{2}\right)^{2}+\left(A^{3}\right)^{2}+\frac{1}{2}\left(A^{4}\right)^{2}+\right. \\
\left.\frac{1}{2}\left(A^{5}\right)^{2}+\frac{1}{2}\left(A^{6}\right)^{2}+\frac{1}{2}\left(A^{7}\right)^{2}+\frac{1}{3}\left(A^{8}\right)^{2}\right],
\end{gathered}
$$

where $m_{g l}^{2} \equiv g^{2} v^{2}$ is the gluon mass parameter of the theory.

After the chosen shifts the following four combinations of scalar fields

$$
\phi_{1}+\frac{\lambda_{3}}{\lambda_{1}+\lambda_{3}} \sigma_{3}, \quad \sigma_{3}, \quad \sigma_{1}+\phi_{3}, \quad \sigma_{2}-\phi_{4}
$$

become massive Higgs particles.

The following eight combinations

$$
\sigma_{1}-\phi_{3}, \phi_{4}+\sigma_{2}, \phi_{2}-\sigma_{4}, \phi_{2}+\sigma_{4}, \phi_{5}, \phi_{6}, \sigma_{5}, \sigma_{6}
$$

become massless Goldstone ghosts.

Now one can make transition to the unitary gauge. All ghost fields as usual disappear from the Lagrangian. Following the approach of [3] one can remove in the unitary gauge all Higgs fields from the Lagrangian preserving on mass-shell renormalizability of the theory.

To give the derivation of this statement let us consuder as an example the simplified case (the generalization to the above case will be straightforward). Let us consider the known model given by the initial $S U(2)$-invariant Lagrangian of interaction of vector bosons and scalar fields possessing the spontaneously broken symmetry

$$
L=-\frac{1}{4} F_{\mu v}^{a} F_{\mu \nu}^{a}+\left(D_{\mu} \Phi\right)^{+} D_{\mu} \Phi-\lambda\left(\Phi^{+} \Phi-v^{2}\right)^{2}
$$

with the doublet of scalar fields $\Phi(x)$ in the fundamental representation of the group.

Here $D_{\mu} \Phi=\left(\partial_{\mu}-i \frac{\tau}{2}^{a} W_{\mu}^{a}\right) \Phi$ is the covariant derivative, $\tau^{a}$ are the Pauli matrices, $\lambda>0$, $v^{2}>0$. (This model can be considered as the Standard Model of electroweak interactions without $U(1)$-interaction and fermions. The derivation given below can be applied also to the complete Standard Model, the $\gamma_{5^{-}}$matrix being treated within dimensional regularization according to the technique of [5].)

To get the complete Lagrangian one makes the shift of the scalar field

$$
\Phi(x)=\frac{1}{\sqrt{2}}\left(\begin{array}{l}
i \phi_{1}(x)+\phi_{2}(x) \\
\sqrt{2} v+\chi(x)-i \phi_{3}(x)
\end{array}\right)
$$


fixes the gauge and adds ultraviolet counterterms. Let us consider two gauges: the widely used $R_{\xi}$-gauge [6], [7] with an arbitrary parameter $\xi$ and the unitary gauge.

In the $R_{\xi}$-gauge one gets the theory described by the Lagrangian

$$
\begin{gathered}
L_{R_{\xi}}=-\frac{1}{4} F_{\mu \nu}^{a} F_{\mu \nu}^{a}+\frac{m^{2}}{2} W_{\mu}^{a} W_{\mu}^{a}-m W_{\mu}^{a} \partial_{\mu} \phi^{a}+\frac{1}{2} \partial_{\mu} \phi^{a} \partial_{\mu} \phi^{a}+\frac{1}{2} \partial_{\mu} \chi \partial_{\mu} \chi \\
-\frac{M^{2}}{2} \chi^{2}+\frac{g}{2} W_{\mu}^{a}\left(\phi^{a} \partial_{\mu} \chi-\chi \partial_{\mu} \phi^{a}+\varepsilon^{a b c} \phi^{b} \partial_{\mu} \phi^{c}\right)+\frac{m g}{2} \chi W_{\mu}^{a} W_{\mu}^{a} \\
+\frac{g^{2}}{8}\left(\chi^{2}+\phi^{a} \phi^{a}\right) W_{\mu}^{2}-\frac{g M^{2}}{4 m} \chi\left(\chi^{2}+\phi^{a} \phi^{a}\right)-\frac{g^{2} M^{2}}{32 m^{2}}\left(\chi^{2}+\phi^{a} \phi^{a}\right)^{2} \\
-\frac{1}{2 \xi}\left(\partial_{\mu} W_{\mu}^{a}+\xi m \phi^{a}\right)^{2} \\
+\partial_{\mu} \bar{c}^{a}\left(\partial_{\mu} c^{a}-g \varepsilon^{a b c} c^{b} W_{\mu}^{c}\right)-\xi m^{2} \bar{c}^{a} c^{a}-\frac{g}{2} \xi m \chi \bar{c}^{a} c^{a}+\frac{g}{2} \xi m \varepsilon^{a b c} \bar{c}^{a} c^{b} \phi^{c} \\
+ \text { counterterms }
\end{gathered}
$$

This theory describes three physical massive vector bosons with the mass $m=g v / \sqrt{2}$, and the physical Higgs field $\chi$ with the mass $M=2 \lambda v$. Here are also Goldstone ghosts $\phi^{a}$ and Faddeev-Popov ghosts $c^{a}$ with masses $\xi m^{2}$. The structure of the counterterms (consistent with gauge invariance and Slavnov-Taylor identities [8, 9] to ensure unitarity) is well known, see e.g. [10].

This is the renormalizable gauge, i.e. Green functions are finite. The corresponding propagators in momentum space are

$$
\begin{gathered}
<T\left(W_{\mu}^{a} W_{v}^{b}\right)>=-i \delta^{a b}\left(\frac{g_{\mu v}-k_{\mu} k_{v} / m^{2}}{k^{2}-m^{2}}+\frac{k_{\mu} k_{v} / m^{2}}{k^{2}-\xi m^{2}}\right) \\
<T\left(\phi^{a} \phi^{b}\right)>=-i \delta^{a b} \frac{1}{k^{2}-\xi m^{2}} \\
<T\left(\bar{c}^{a} c^{b}\right)>=-i \delta^{a b} \frac{1}{k^{2}-\xi m^{2}} \\
<T(\chi \chi)>=-i \frac{1}{k^{2}-M^{2}}
\end{gathered}
$$

In the unitary gauge defined by the gauge condition $\phi^{a}=0$ one has the Lagrangian

$$
\begin{gathered}
L_{U}=-\frac{1}{4} F_{\mu \nu}^{a} F_{\mu \nu}^{a}+\frac{m^{2}}{2} W_{\mu}^{a} W_{\mu}^{a}+\frac{1}{2} \partial_{\mu} \chi \partial_{\mu} \chi-\frac{M^{2}}{2} \chi^{2} \\
+\frac{m g}{2} \chi W_{\mu}^{a} W_{\mu}^{a}+\frac{g^{2}}{8} \chi^{2} W_{\mu}^{a} W_{\mu}^{a}-\frac{g M^{2}}{4 m} \chi^{3}-\frac{g^{2} M^{2}}{32 m^{2}} \chi^{4}+\text { counterterms }
\end{gathered}
$$

The propagators in the unitary gauge are obtained from those of the $R_{\xi}$-gauge in eq.(17) by taking the limit $\xi \rightarrow \infty$. The theory in the unitary gauge is renormalizable only on mass-shell, i.e. Green functions are divergent at $\varepsilon \rightarrow 0$ but the S-matrix elements are finite. In this gauge all unphysical particles (longitudinal quanta of vector fields and ghosts) are absent and unitarity of the theory is manifest. 
To consider renormalization for our purpose it is convenient to use the Bogoliubov-ParasiukHepp subtraction scheme [11]. As it is well known in this scheme a counterterm of e.g. a primitively divergent Feynman diagram is the truncated Taylor expansion of the diagram itself at some fixed values of external momenta. Hence counterterms of mass dependent diagrams are also mass dependent. Needless to say that subtractions should respect Slavnov-Taylor identities.

Let us consider S-matrix elements in the $R_{\xi}$-gauge without external Higgs bosons (i.e. with external W-bosons only in this simplified model). We will analyze the dependence of diagrams on the Higgs mass $M$ by using for convinience the expansion in large $M$ (after renormalization but before the removing regularization). The algorithm for the large mass expansion of Feynman diagrams is given e.g. in [12] (where it is quite reasonably checked in calculations of the 4-loop diagrams for the Z-boson decay into hadrons). It can be rigorously derived with the technique of [13].

We separate all diagrams into physical ones which are not nullified in the limit $\xi \rightarrow \infty$ and unphysical ones which are nullified. In this limit the propagator of the W-boson reduces to the known unitary form

$$
\begin{aligned}
\lim _{\xi \rightarrow \infty}<T\left(W_{\mu}^{a} W_{v}^{b}\right)>= & -i \delta^{a b} \lim _{\xi \rightarrow \infty}\left(\frac{g_{\mu v}-k_{\mu} k_{v} / m^{2}}{k^{2}-m^{2}}+\frac{k_{\mu} k_{v} / m^{2}}{k^{2}-\xi m^{2}}\right)= \\
& =-i \delta^{a b} \frac{g_{\mu v}-k_{\mu} k_{v} / m^{2}}{k^{2}-m^{2}}
\end{aligned}
$$

The propagators of the Goldstone bosons $\phi^{a}$ and ghosts $c^{a}$ vanish in this limit and correspondingly all diagrams which contain these propagators are also nullified. The limit is a little bit subtle for diagrams containing ghosts loops coupled to the Hogs boson since the corresponding coupling constant itself contains the parameter $\xi$ in the Lagrangian, see eq.(16). For example in the one-loop case one gets

$$
\lim _{\xi \rightarrow \infty} \xi^{2} \int d^{d} p \frac{1}{\left(p^{2}-\xi m^{2}\right)\left((p+q)^{2}-\xi m^{2}\right)}=\int d^{d} p=0
$$

where zero is obtained due to the famous property of dimensional regularization to nullify scaleless integrals. The limit $\xi \rightarrow \infty$ commutes with integrations in Feynman integrals within dimensional regularization.

Thus in our notations the physical diagrams are the diagrams which do not contain Goldstone bosons propagators or ghosts propagators and the unphysical diagrams are the diagrams which contain such propagators.

Within the large-M expansion the physical diagrams with $\chi$-propagators contain either terms with integer negative powers of $M^{2}$

$$
\frac{1}{M^{2 n}}, \quad n=1,2,3, \ldots
$$

or terms with non-integer powers of $M^{2}$ (non-integer powers contain $\varepsilon$ )

$$
\frac{1}{M^{2(k+l \varepsilon)}}, \quad k \text {-integer, } \quad l \text {-positive integer }
$$

This is because each vertex with the large factor $M^{2}$ has three or four attached $\chi$-propagators due to the structure of the Higgs boson self-coupling. 
In contrast, unphysical diagrams can have polynomial in $M$ terms due to the four- $\phi$ vertex with the large factor $M^{2}$. But they are $\xi$-dependent (they are nullified in the limit $\xi \rightarrow \infty$ ) and this polynomial terms cancel in S-matrix elements.

In the renormalizable $R_{\xi}$-gauge one can present ultraviolet renormalization in a standard form of the Bogoliubov-Parasiuk R-operation for individual diagrams. This ensures that after renormalization the $M$-dependent terms are finite at $\varepsilon \rightarrow 0$ separately from $M$-independent terms. Thus if one removes all $M$-dependent terms one is left with a finite expression.

On the Lagrangian level it means in the unitary gauge that one removes from $L_{U}$ all terms containing the field $\chi$ and also all $M$-dependent terms in the counterterms. This should be done in the unitary gauge because in the $R_{\xi}$-gauge some diagrams containing propagators of Higgs particle can give contributions not depending on the Higgs mass $M$. In contrast in the unitary gauge all diagrams containing Higgs propagators give only contributions depending on $M$ so there is one to one correspondence between $M$-dependent diagrams and terms in the Lagrangian containing the Higgs field $\chi$

The resulting theory is on mass-shell finite. This is the massive Yang-Mills theory

$$
L_{Y M}=-\frac{1}{4}\left(\partial_{\mu} W_{v}^{a}-\partial_{v} W_{\mu}^{a}+\frac{z_{1}}{z_{2}} g f^{a b c} W_{\mu}^{b} W_{v}^{c}\right)^{2}+m^{2} W_{\mu}^{a} W_{\mu}^{a}+\text { counterterms. }
$$

Thus the Higgs mechanism can be considered as an efficient mathematical tool to observe on mass-shell renormalizability of the massive Yang-Mills theory which is far from to be obvious directly.

It is known that the Higgs theories of vector mesons posses so called tree level unitarity, see e.g.[14],[15], [16] and references therein. Tree level cross sections of such theories grow at high energies slowly enough and do not exceed the so called unitary limit imposed by the unitarity condition. The reversed statement is also proved: from the condition of tree level unitarity follows that a theory of vector mesons should be a Higgs theory [15]. But one can see that tree level unitarity is not the necessary condition for renormalizability. Tree level unitarity is violated in the massive Yang-Mills theory. It indicates that higher order contributions become relevant at high energies and one looses the perturbative control over the theory. This is due to the presence in external states of S-matrix elements of longitudinally polarised $W$-bosons which wave functions grow with energy like $E / m$ where $E$ is typical energy of the process. But in the physical $S U(2) \times U(1)$ theory with inclusion of fermions the massive gauge bosons are highly unstable particles which do not appear as external states of S-matrix elements in complete calculations and correspondingly tree level unitarity is present (with or without the Higgs boson).

Let us now return to our Lagrangian (10) with two scalar triplets which after spontaneous symmetry breaking has four Higgs particles (13). Following the above approach we can remove in the unitary gauge all four Higgs fields from the Lagrangian preserving on mass-shell renormalizability of the theory. The Lagrangian of the resulting QCD with massive gluons is

$$
L_{\text {massive } Q C D}=L_{M}-\frac{1}{4} F_{\mu \nu}^{a} F_{\mu \nu}^{a}+i \bar{\psi}_{f} \gamma_{\mu} D_{\mu} \psi_{f}-m_{f} \bar{\psi}_{f} \psi_{f}+\text { counterterms }
$$

where $L_{M}$ is given in eq.(12).

Let us note that on mass-shell renormalizability does not mean that one should consider quarks and gluons as free external particles. It means that in the $S U(3) \times S U(2) \times U(1)$ theory the $S$-matrix elements with the physical external particles will be finite. 
One can calculate the one-loop $\beta$-function in this theory to obtain for a massless renormalization scheme (i.e. a scheme where renormalization group functions do not depend on masses, e.g. the MS-scheme) the following result

$$
\begin{gathered}
\beta\left(a_{s}\right)=\mu^{2} \frac{\partial a_{s}}{\partial \mu^{2}}=\sum_{i \geq 0} \beta_{i} a_{s}^{i+2}, \\
\beta_{0}=-\frac{7}{2} C_{A}+\frac{4}{3} T_{F} n_{f},
\end{gathered}
$$

here $C_{A}=3$ is the Casimir operator of the adjoint representation of the $S U(3)$ color group, $T_{F}=1 / 2$ is the trace normalization of the fundamental representation, $n_{f}$ is the number of active quark flavors.

Thus asymptotic freedom remains valid in the considered theory with massive gluons.

Acknowledgments. The author is grateful to collaborators of the Theory division of INR for helpful discussions. The work is supported in part by the grant for the Leading Scientific Schools NS-5590.2012.2.

\section{References}

[1] D.J. Gross, F. Wilczek, Phys. Rev. Lett. 30 (1973) 1343;

H.D. Politzer, Phys. Rev. Lett. 30 (1973) 1346.

G. 't Hooft, report at the Marseille Conference on Yang-Mills Fields, 1972.

[2] G. Källen, Helv. Phys. Acta 25 (1952) 417; H. Lehmann, Nuovo Cim. 11 (1954) 342.

[3] S.A. Larin, e-Print: hep-ph/0503198; Phys.Part.Nucl. 44 (2013) 386.

[4] P.W. Higgs, Phys. Lett. 12 (1964) 132.

F.Englert and R. Brout, Phys. Rev. Lett. 13 (1964) 321.

[5] S.A. Larin, Phys. Lett. B 303 (1993) 113; e-Print: hep-ph/9302240.

[6] G. 't Hooft, Nucl. Phys. B 35 (1971) 167.

[7] K. Fujikawa, B.W. Lee and A.I. Sanda, Phys. Rev. D6 (1972) 2923.

[8] A.A. Slavnov, Theor. Math. Phys. 10 (1972) 99.

[9] J.C. Taylor, Nucl. Phys. B 33 (1971) 436.

[10] L.D. Faddeev and A.A. Slavnov, Gauge fields. Introduction to quantum theory, Front. Phys. 83 (1990) 1.

[11] N.N. Bogoliubov and O.S. Parasiuk, Acta Math. 97 (1957) 227.

K. Hepp, Comm. Math. Phys. 2 (1966) 301.

[12] S.A. Larin, T. van Ritbergen and J.A.M. Vermaseren, Nucl. Phys. B 438 (1995) 278.

[13] S.A. Larin, Phys. Lett. B 469 (1999) 220.

[14] C.H. Llewellyn Smith, Phys. Lett. 46 B (1973) 233.

[15] J.M. Cornwall, D.N. Levin and G. Tiktopoulos, Phys. Rev. D 10 (1974) 1145.

[16] J.C. Taylor, Gauge theories of weak interactions, Cambridge University Press, 1976. 